\title{
In silico Study of the Dynamic Interaction between Extracorporeal Circulation and Native Circulation
}

Markus Bongert, University of Applied Sciences and Arts, Research Center for BioMedical Technology (BMT), Dortmund, Germany, bongert@fh-dortmund.de

Johannes Gehron, University Hospital Gießen and Marburg, Cardiovascular Surgery, Gießen, Germany, Johannes.Gehron@chiru.med.uni-giessen.de

Marius Geller, University of Applied Sciences and Arts, Research Center for BioMedical Technology (BMT), Dortmund, Germany, geller@fh-dortmund.de

Andreas Böning, University Hospital Gießen and Marburg, Cardiovascular Surgery, Gießen, Germany, Andreas.Boening@chiru.med.uni-giessen.de

Philippe Grieshaber, University Hospital Gießen and Marburg, Cardiovascular Surgery, Gießen, Germany, Philippe.Grieshaber@chiru.med.uni-giessen.de

An in silico investigation of modelled Extracorporeal Life Support (ECLS) via a femoral arterial cannula revealed the existence of both a defined separation zone between the opposing flows (ECLS, native flow) and different ranges dependent on flow distribution. The interaction between pulsating native circulation and constant ECLS flow is dynamic. A transient simulation model was developed to investigate the dynamic influence on this fluid mechanical interaction.

The in silico model is based on a CT-generated 3D model derived from a life-sized silicon aorta. A geometric standard cannula $(16 \mathrm{Fr})$ is inserted femoral. Inlet boundary conditions such as the temporal flow profile of a subject from the left ventricle (native circulation) and the flow from the femoral cannula are varied such that during transient simulations the summed flow (total perfusion) is $5.5 \mathrm{l} / \mathrm{min}$. The outlet pressure boundary conditions at the branching arteries are selected such as to model the downstream vascular system.

Transient simulations revealed the dynamic effects of different flow fractions (Heart - ECLS) on the flow. Stationary simulations show a separation zone between the two flows, the position of which respectively the ECLS-range, oscillates dependent of the native circulation. Furthermore, it was noted that a raised pulse was impedimental to ECLS. This can be partly compensated by increasing the length of cannula inserted. At the same time the ECLS supply for the brain can improve at the cost of performance post-bifurcation. Increasing the ECLS fraction to above 50\% flow led to retrograde flow combined with blood suction from the femoral artery.

The EMPAC project model has been further developed to include investigation of the dynamic effects of blood flow. This has made it possible for the first time to analyse in detail and evaluate the temporal effects of both opposing flows streams. A subsequent investigation explains whether aortic elasticity plays a significant role. 


\section{Comparison of two biological Aortic Valve Prostheses inside patient-specific Aorta Model by bi-directional Fluid-Structure Interaction}

Markus Bongert, University of Applied Sciences and Arts, Research Center for BioMedical Technology (BMT), Dortmund, Germany, bongert@fh-dortmund.de

Jan Wüst, University of Applied Sciences and Arts, Research Center for BioMedical Technology (BMT), Dortmund, Germany, jan.wuest@fh-dortmund.de

Marius Geller, University of Applied Sciences and Arts, Research Center for BioMedical Technology (BMT), Dortmund, Germany, geller@fh-dortmund.de

Markus Schlömicher, Ruhr-University Bochum, University Medical Center Bergmannsheil, Clinic for Cardiosurgery and Thoracic Surgery, Bochum, Germany, markus.schloemicher@bergmannsheil.de

Tim Ricken, University Stuttgart, Institute for Mechanics, Structural Analysis and Dynamics, Stuttgart, Germany, tim.ricken@isd.uni-stuttgart.de

Volkmar Nicolas, Ruhr-University Bochum, University Medical Center Bergmannsheil, Institute for Radiological Diagnostics, Interventional Radiology and Nuclear Medicine, Bochum, Germany, volkmar.nicolas@bergmannsheil.de

Justus Strauch, Ruhr-University Bochum, University Medical Center Bergmannsheil, Clinic for Cardiosurgery and Thoracic Surgery, Bochum, Germany, justus.strauch@bergmannsheil.de

In Germany in 2016 17,085 patients received TAVI operations and 9,579 had conventional aortic valve surgery. The 'Heart Team' uses established scoring systems (EuroSCORE, STS, German AV Score) to evaluate operation risks and which technique to use. However, such risk grading fails to consider patient morphology and possible long-term behaviour of the replacement valve chosen. Therefore, pre-operative simulation of the dynamic loading on the valve leaflets after TAVR provides information vital for the selection of the appropriate aortic valve therapy - interventional versus conventional.

Individual aorta used in this study was captured by MRI. Segmentation and data processing were done with Mimic Innovation Suite. The available biological aortic valves prostheses were reverse engineered to create a 3D CAD model. Simulations combined bi-directional fluid structure interaction (FSI) with a first order Ogden model of the hyperelastic behaviour of aortic leaflets from bovine pericardium.

Movements induced by flow and the resultant tension on the biological leaflets were computed with developed simulation model. Stress analyses of the leaflets showed behaviour attributable to their particular structure. Both valves showed two stress peaks within the initial $0.3 \mathrm{~s}$. Maximum stress occurred, however, at other time points. Furthermore, the initial increase in stress showed a delayed onset. The patterns of movement were also significantly different. So, at opening of the valve, the freely perfused area of the valve, the freedom of leaflet movement and symmetry at closure were different in the two valves.

Simulated movement of valve leaflets corresponds well with reality. The estimated stresses clearly lie below thresholds published in the literature for bovine pericardium. It is planned to further develop the current workflow to increase stability and optimize processing time, with the intention of providing the 'Heart Team' with a tool for incorporating individual anatomy when selecting the aortic valves. 


\section{Performance of Stereo Matching Algorithms in 3D Endoscopy}

Dennis Schuldt, Research Center for BioMedical Technology (BMT), University of Applied Sciences and Arts, Dortmund, Germany, dennis.schuldt@fh-dortmund.de

Fatih Tanriverdi, Research Center for BioMedical Technology (BMT), University of Applied Sciences and Arts, Dortmund, Germany, fatih.tanriverdi@,fh-dortmund.de

Jörg Thiem, Research Center for BioMedical Technology (BMT), University of Applied Sciences and Arts, Dortmund, Germany, joerg.thiem@fh-dortmund.de

For the task of in-vivo 3D reconstruction from stereo image pairs of a binocular endoscope in MIS, the question has to be answered, how different approaches with classical matching algorithms affect the measurement accuracy. We consider the medical scenario of an endometriosis intervention. In addition, we have to discuss methods to optimize and improve the accuracy in medical image data.

To evaluate the measurement error, we use a known target pattern under ideal lighting conditions. The relative spatial relationship of all target points is well-known.

The real clinical data is based on a set of acquired stereo videos during a laparoscopic intervention for endometriosis extraction (object diameter approx. $3 \mathrm{~mm}$ ). The set of 174 stereo frames is manually annoted with ground truth information, i.e. the locations of two measuring points in both left and right views of each stereo frame. These points characterize the size of the endometriosis area (longest side) and the task of the benchmark is to measure the size in metrical units.

We consider several classic similarity metrics for the matching process, e.g. the ZSAD (zero mean sum of absolute differences)

the ZSSD (zero mean sum of squared differences)

$$
s\left(I_{1}, I_{2}\right)=\sum_{(u, v) \in I}\left|\left(I_{1}[u, v]-\bar{I}_{1}\right)-\left(I_{2}[u, v]-\bar{I}_{2}\right)\right|
$$

$$
s\left(I_{1}, I_{2}\right)=\sum_{(u, v) \in I}\left(\left(I_{1}[u, v]-\bar{I}_{1}\right)-\left(I_{2}[u, v]-\bar{I}_{2}\right)\right)^{2}
$$

and the ZNCC (zero mean normalized cross correlation)

$$
s\left(I_{1}, I_{2}\right)=\frac{\sum_{(u, v) \in I}\left(I_{1}[u, v]-\bar{I}_{1}\right) \cdot\left(I_{2}[u, v]-\bar{I}_{2}\right)}{\sqrt{\sum_{(u, v) \in I}\left(I_{1}[u, v]-\bar{I}_{1}\right)^{2} \cdot \sum_{(u, v) \in I}\left(I_{2}[u, v]-\bar{I}_{2}\right)^{2}}}
$$

where $I_{1,2}$ is the image pixel data in a template window and $\bar{I}_{1,2}$ the average value in the window.

The winner of the matching algorithm results in the horizontal offset of a point of interest between the left and right image planes, i.e. the disparity value $d=u_{1}-u_{2}$, which is used to reconstruct the $3 \mathrm{D}$ coordinate $P=(x, y, z)^{T}$ w.r.t. the left camera. This is done with both measuring points $P_{1,2}$ and we can therefore calculate the euclidean distance between the points $D=\left\|P_{1}-P_{2}\right\|_{2}$. We can estimate the error, because we know the exact distance in the given target. In the medical data we compare the distance that results from the matching process with the distance based on the ground truth data.

The results show that the accuracy highly depends on the chosen metric, the ideal target pattern results have an average error of $|\mu|=0,55 \mathrm{~mm}$ with $\sigma=0,25 \mathrm{~mm}$. In comparison, for the medical image data we get the best average error with ZNCC and a degradation by a factor of $>3$. Therefore, to overcome the challenging uncooperative medical data, we investigated how the algorithms can be adapted and how the raw image data can be preprocessed to improve the results. 


\section{Feature Matching Improvements Based on Hyperspectral Imaging}

Fatih Tanriverdi, Research Center for BioMedical Technology (BMT), University of Applied Sciences and Arts, Dortmund, Germany, fatih.tanriverdi@fh-dortmund.de

Dennis Schuldt, Research Center for BioMedical Technology (BMT), University of Applied Sciences and Arts, Dortmund, Germany, dennis.schuldt@fh-dortmund.de

Jörg Thiem, Research Center for BioMedical Technology (BMT), University of Applied Sciences and Arts, Dortmund, Germany, joerg.thiem@fh-dortmund.de

Stereo instrument is an often used image based procedure in medical applications that currently does not provide any assisting feature. Targeted use of stereo technology could make assistance systems available to medical users. An essential component of this is the correspondence analysis, which forms the basis for a $3 \mathrm{D}$ reconstruction. In order to create a dense depth map, features must be found in both images of the stereo pair and assigned to one another with a matching score. Limitations in medical in vivo image data are often reflections of the tissue surface due to the fluid film and fast movements of the medical imaging tool. These limitations lead to gaps in the depth map and misinformation in the 3D reconstruction. In this work, we focus on removing these constraints. Therefore, it is necessary to develop an applicable descriptor with the aid of a hyperspectral camera. Feature matching is often only done in grayscale converted RGB images. Hyperspectral images are a composite of several grayscale images that correspond to different wavelengths, in this study between $470 \mathrm{~nm}$ and $620 \mathrm{~nm}$. Under laboratory conditions, a HSI descriptor will be developed based on nonmedical data. The higher information content of these HSI cubes $(256 \times 512 \times 16$ pixels $)$ let expect to reach robust features and better matching results compared to grayscale image matching which will be evaluated in this study. By selective thresholding multiple bands are used to match features. Thus, bands that do not provide information can be discarded and matching will be done with the valid bands only. This is not possible in the case of conventional RGB camera sensors, as the interference affects all three bands. After final investigations under laboratory conditions, the HSI descriptor has to be tested and evaluated with medical ex-vivo images, which will be taken in clinical studies at the Endometriosis Centre of Marienkrankhaus in Schwerte/Germany. 


\section{Freehand 3D ultrasound visualization of vessels - First results of an augmented reality approach}

Christopher Andreas Nielsen, Medical Engineering Laboratory, Westfälische Hochschule, University of Applied Sciences, Gelsenkirchen, Germany, christopher.nielsen@studmail.w-hs.de

Heinrich Martin Overhoff, Medical Engineering Laboratory, Westfälische Hochschule, University of Applied Sciences, Gelsenkirchen, Germany, martin.overhoff@w-hs.de

Stefan Maas, SomaView GbR, Bochum, Germany, stefan.maas@somaview.glass

About two millionen central venous catheters (CVCs) are laid every year in Germany. Ultrasound imaging is the standard modality for visualizing vessels and catheter needle during the insertion of a CVC. Studies report complications during or after the insertion of CVCs from 5 to $20 \%$, but additionally a reduced complication rate with improved insertion experience.

To help especially unexperinced physicians, an augmented reality (AR) based ultrasound system was developed. The system allows the physician to see the live ultrasound image directly beneath the transducer by using AR-glasses. Additonaly it is possible to realize a freehand 3D sweep to visualize up to 100 slices over a range up to $15 \mathrm{~cm}$ inside the region of interest. The slices are visualized inside the AR-glasses and can be examined as a virtual "pseudo 3D volume" exactly at the position of the "real" patients anatomy.

The system allows the usage of simple image processing filters to enhance the visualization of the "pseudo 3D volume" in real time.

The system consists of a clinical ultrasound device with a linar transducer, a standard PC, AR-glasses (ODG R-7) and the developed software. The software was implemented with Unity Personal (version 5.2.3f1), Vuforia (version 5.0.6) and Visual Studio 2010 Professional.

Ultrasound images are collected via the DVI-port of the ultrasound device and are send to the AR-glasses via WiFi. An optical marker attached to the transducer allows to track its position and to visualize the ultrasound image directly beneath the transducer when looking through the AR-glasses. A second marker is used as a reference for the "pseudo 3D volume".

The visualization of the "pseudo 3D volume" enables a first impression of the region of interest. To improve the visualization quality, future development addresses its conversion into a regular volume for the purposes of volume raycasting. 


\section{Flexible interventional imaging system based on miniaturized $X$-ray tubes (FlexScan)}

Sinja Lagotzki, Institute of medical technology, INKA, Otto-von-Guericke University, Magdeburg, Germany, sinja.lagotzki@gmx.de

Muhammad Usama Iftikar, Institute of medical technology, INKA, Otto-von-Guericke University, Magdeburg, Germany, muhammad.iftikhar@st.ovgu.de

Michael Friebe Institute of medical technology, INKA, Otto-von-Guericke University, Magdeburg, Germany, michael.friebe@ovgu.de

Axel Boese, Institute of medical technology, INKA, Otto-von-Guericke University, Magdeburg, Germany, axel.boese@ovgu.de

In orthopedic hand surgeries C-arms are the standard imaging modalities for procedure and tool guidance. However, the currently used systems have a large footprint and high weight, which can lead to workspace restrictions and difficult positioning of the device at the desired imaging position. The aim of this paper is to present a prototype of a new, flexible, lightweight and small footprint X-ray system, which is capable to create 2D projection images from different orientations. The new design includes a miniaturized X-ray tube covered in a custom made case mounted on a flexible holding arm attached to the standard OR table rail. With that, fast positioning and fixation for the subsequent image acquisition is achieved. A flat panel detector is placed in an adjustable metal sheet construction below the table. For safety aspects an overlay of the X-ray cone beam with an integrated light source visualizes the irradiation area. The acquired images are visualized on a 2-in-1 netbook. A foot pedal initiates the imaging process. A prototype of the free movable miniaturized X-ray system FlexScan was build. Workspace restriction, usability and general imaging needs were simulated and tested. FlexScan has the potential to improve X-ray guided interventions on extremities especially for small private surgery centers. It fulfills the general imaging needs and is capable of producing 2D projection images from different orientations within a small and lightweight setup. 